\title{
Classical and quantum analysis of a heterotriatomic molecular Bose-Einstein-condensate model
}

\author{
A. P. Tonel, ${ }^{1}$ C. C. N. Kuhn, ${ }^{2}$ G. Santos, ${ }^{3}$ A. Foerster, ${ }^{2}$ I. Roditi, ${ }^{4}$ and Z. V. T. Santos ${ }^{4}$ \\ ${ }^{1}$ CCET da Unipampa, Bagé, RS, Brazil \\ ${ }^{2}$ Instituto de Física-UFRGS, Porto Alegre, RS, Brazil \\ ${ }^{3}$ Departamento de Física-UFS, São Cristóvão, SE, Brazil \\ ${ }^{4}$ Centro Brasileiro de Pesquisas Físicas-CBPF, Rio de Janeiro, Brazil
}

(Received 5 November 2008; published 26 January 2009)

\begin{abstract}
We investigate an integrable Hamiltonian modeling a heterotriatomic molecular Bose-Einstein condensate. This model describes a mixture of two species of atoms in different proportions, which can combine to form a triatomic molecule. Beginning with a classical analysis, we determine the fixed points of the system. Bifurcations of these points separate the parameter space into different regions. Three distinct scenarios are found, varying with the atomic population imbalance. This result suggests the ground-state properties of the quantum model exhibit a sensitivity on the atomic population imbalance, which is confirmed by a quantum analysis using different approaches, such as the ground-state expectation values, the behavior of the quantum dynamics, the energy gap, and the ground-state fidelity.
\end{abstract}

DOI: 10.1103/PhysRevA.79.013624

PACS number(s): 03.75.Hh, 02.30.Ik

\section{INTRODUCTION}

The experimental achievement that led to the BoseEinstein condensates (BECs), using dilute alkali gases at ultracold temperatures $[1,2]$, induced a substantial effort dedicated to the understanding of new properties of BECs. In particular, the development of the techniques used in the production and manipulation of ultracold atoms and molecules [3] has opened the way to another field, the "chemistry" of ultracold systems, i.e., where the atomic constituents of the dilute gas may recombine forming molecules. Such molecular BEC compounds have been obtained by different techniques [4], for instance, by Feshbach resonances [5-7] or photoassociation [8]. There can also occur atom-molecule interactions that must be at least three body in nature [9], bringing up a stimulus and challenges to our physical understanding. Experimental evidence for three-body recombinations [10] as well as for Efimov states [11] provides a physical ground and stimulus for the search of triatomic molecular BECs and for the investigation of their theoretical aspects, which is our main interest.

From the theoretical point of view, ultracold atomic and molecular systems are characterized by their large quantum fluctuations. In this sense, the search for exactly solvable models describing atomic and molecular BEC becomes relevant. Indeed this has become a very active field of research [12-20], and the experimental relevance of these models is currently a very active research subject [21]. Those solvable models are expected to provide a significant impact in this area, a view that has been promoted in $[22,23]$. Increasing evidence and recent results show that multiatomic systems may be interesting and relevant for ultracold atomicmolecular Bose-Einstein condensates. A significant question in this context is whether more complex ultracold molecules could be created than simple dimers [24]. Also, due to the more sophisticated nature of the control of the interatomic interactions, in the case of triatomic molecules, one expects a rich quantum phase structure. Indeed, very recent experimental results confirm the existence of heteroatomic bosonic tri- mers in ultracold mixtures [25], which provide us with additional motivation to pursue the present investigation.

In this paper we analyze an integrable model describing a heterotriatomic molecular Bose-Einstein condensate where atomic BECs can combine (in different proportions) to produce a compound with two atoms of the same kind and a third one of a different species. Our model, which has been shown to be solvable in [26], includes besides the interconversion of atoms to molecules and vice versa, a linear interaction corresponding to the external potential and a bilinear interaction corresponding to the scattering between atomsatoms, atoms-molecules, and molecules-molecules. We start our analysis of this model by a classical treatment where we obtain its phase space determining, in particular, the fixed points. We find that for certain coupling parameters bifurcation of the fixed points occurs, and we can determine a parameter space diagram, which classifies the found fixed points. This diagram is determined for the imbalance of the number of atoms, which allows us to classify it in three distinct cases. Specifically, when the imbalance is equal to zero or is negative there is a spontaneous appearance of additional boundaries in the parameter space (one for the zero case and two for the negative case), some of which can be identified with bifurcations of the minimum of the classical Hamiltonian. We also perform a quantum analysis, where we study the quantum dynamics and make a comparison with the classical results. Here we are interested in studying the ground state of the model, because as actual systems are in ultracold temperature, some insight can be obtained from the ground state. Furthermore, as pointed out before, the presence of large quantum fluctuations makes it interesting to look for the phase structure at zero temperature, the quantum phase transitions. In our case we are able to look for signatures of quantum phase transition. Here we use two definitions: energy gap and ground-state fidelity in order to find a quantum phase pretransition, a term that will be explained later. We observe that the critical points are pinned down in complete agreement with the classical analysis.

The paper is organized as follows. In Sec. II we present our integrable model. Section III is devoted to the classical 
analysis where the parameter diagram is obtained. Section IV is devoted to the quantum analysis where we show the quantum dynamics and a study about quantum phase pretransition. Section V is devoted to our conclusions.

\section{MODEL}

Let us consider the following Hamiltonian describing a heterotriatomic molecular Bose-Einstein condensate with two identical species of atoms, denoted by $a$, which can be combined with a different type of atom, denoted by $b$, to produce a molecule labeled by $c$. In terms of canonical creation and annihilation operators $\left\{a, b, c, a^{\dagger}, b^{\dagger}, c^{\dagger}\right\}$ satisfying the usual commutation relations $\left[a, a^{\dagger}\right]=I$, etc., the Hamiltonian reads

$$
\begin{aligned}
H= & U_{a a} N_{a}^{2}+U_{b b} N_{b}^{2}+U_{c c} N_{c}^{2}+U_{a b} N_{a} N_{b}+U_{a c} N_{a} N_{c} \\
& +U_{b c} N_{b} N_{c}+\mu_{a} N_{a}+\mu_{b} N_{b}+\mu_{c} N_{c}+\Omega\left(a^{\dagger} a^{\dagger} b^{\dagger} c+c^{\dagger} b a a\right) .
\end{aligned}
$$

The parameters $U_{i j}$ describe $S$-wave scattering, $\mu_{i}$ are external potentials, and $\Omega$ is the amplitude for interconversion of atoms and molecules. $N_{i}$ are the number operators, i.e., $N_{a}$ $=a^{\dagger} a$ is the number of atoms type $a, N_{b}=b^{\dagger} b$ is the number of atoms type $b$, and $N_{c}=c^{\dagger} c$ is the number of molecules.

The Hamiltonian acts on the Fock space spanned by the (unnormalized) vectors

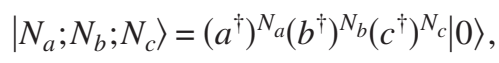

where $|0\rangle$ is the Fock vacuum.

The Hamiltonian above has two independent conserved quantities

$$
N=N_{a}+N_{b}+3 N_{c}, \quad J=N_{a}-2 N_{b},
$$

where $N$ is the total number of atoms and $J$ is the atomic imbalance. It is convenient to introduce $k=J / N$ as the fractional atomic imbalance. Since there are three degrees of freedom and three conserved quantities, the model is integrable. More details about the integrability of this model, using the Bethe ansatz method, can be found in [26]. In what follows we will investigate this model in detail. Below we begin with a classical analysis of the model and determine the fixed points of the system.

\section{CLASSICAL ANALYSIS}

Let $N_{j}, \theta_{j}, j=a, b, c$, be quantum variables satisfying the canonical relations

$$
\left[\theta_{j}, \theta_{k}\right]=\left[N_{j}, N_{k}\right]=0, \quad\left[N_{j}, \theta_{k}\right]=i \delta_{j k} I .
$$

We make a change of variables from the operators $j, j^{\dagger}, j$ $=a, b, c$, to a number-phase representation through

$$
j=\exp \left(i \theta_{j}\right) \sqrt{N_{j}}, \quad j=a, b, c,
$$

such that the canonical commutation relations are preserved. We perform an additional change of variables,

$$
z=\frac{1}{N}\left(N_{a}+N_{b}-3 N_{c}\right),
$$

$$
\theta=\frac{N}{6}\left(2 \theta_{a}+\theta_{b}-\theta_{c}\right),
$$

such that $z$ and $\theta$ are canonically conjugate variables, i.e.,

$$
[z, \theta]=i I
$$

In the limit of large $N$ we can approximate the (rescaled) Hamiltonian by

$$
\begin{aligned}
H= & \frac{4 \Omega N^{2}}{36}\left[\lambda z^{2}+2(\alpha-\lambda) z+\beta\right. \\
& \left.+\left(z+c_{+}\right) \sqrt{\left(z+c_{-}\right)(1-z)} \cos \left(\frac{6 \theta}{N}\right)\right],
\end{aligned}
$$

where we have defined

$$
\begin{gathered}
\lambda=\Delta\left(4 U_{a}+U_{b}+U_{c}+2 U_{a b}-2 U_{a c}-U_{b c}\right), \\
\alpha=\Delta\left[4\left(c_{+}+1\right) U_{a}+\left(c_{-}+1\right) U_{b}+\left(c_{+}+c_{-}+2\right) U_{a b}\right. \\
\left.-\left(1+c_{+}\right) U_{a c}-\left(1+c_{-}\right) \frac{U_{b c}}{2}+\frac{3}{N}\left(2 \mu_{a}+\mu_{b}-\mu_{c}\right)\right], \\
\beta=\Delta\left[4 U_{a} c_{+}^{2}+U_{b} c_{-}^{2}+U_{c}+2 U_{a b} c_{+} c_{-}+2 U_{a c} c_{+}+U_{b c} c_{-}\right. \\
\left.+\frac{6}{N}\left(2 \mu_{a} c_{+}+\mu_{b} c_{-}+\mu_{c}\right)\right],
\end{gathered}
$$

with

$$
\begin{gathered}
c_{-}=1-2 k, \quad c_{+}=1+k, \quad \Delta=\frac{1}{4 \Omega}, \\
k=\frac{J}{N}, \quad k \in[-2,1] .
\end{gathered}
$$

We now regard Eq. (3) as a classical Hamiltonian and investigate the fixed points of the system. The first step is to find Hamilton's equations of motion, which yield

$$
\begin{aligned}
\frac{d z}{d t} & =\frac{\partial H}{\partial \theta}=-\frac{4 \Omega N}{6}\left(z+c_{+}\right) \sqrt{\left(z+c_{-}\right)(1-z)} \sin \left(\frac{6 \theta}{N}\right) \\
\frac{d \theta}{d t}= & -\frac{\partial H}{\partial z}=\frac{4 \Omega N^{2}}{36}[2 \lambda z+2(\alpha-\lambda) \\
& +\frac{2\left(z+c_{-}\right)(1-z)+\left(z+c_{+}\right)(1-z)-\left(z+c_{+}\right)\left(z+c_{-}\right)}{2 \sqrt{\left(z+c_{-}\right)(1-z)}} \\
& \left.\times \cos \left(\frac{6 \theta}{N}\right)\right] .
\end{aligned}
$$

The fixed points of the system are determined by the condition 


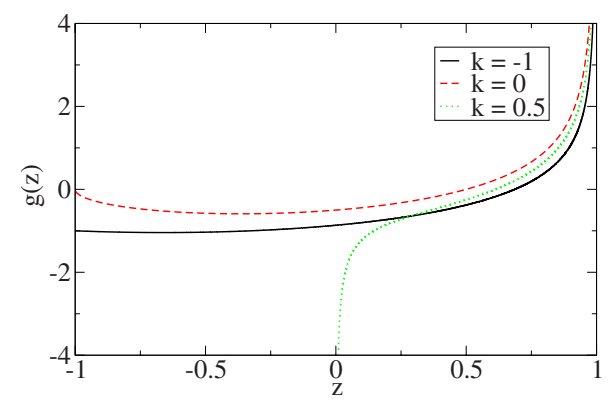

FIG. 1. (Color online) The behavior of the function $g(z)$ for three different values of $k$.

$$
\frac{\partial H}{\partial \theta}=\frac{\partial H}{\partial z}=0
$$

Due to periodicity of the solutions, below we restrict ourselves to $\theta \in[0, N \pi / 3]$. It is convenient to define the functions as follows:

$$
\begin{gathered}
f(z)=\lambda z+\alpha-\lambda, \\
g(z)=-\frac{2(1-z)\left(z+c_{-}\right)+(1-z)\left(z+c_{+}\right)-\left(z+c_{+}\right)\left(z+c_{-}\right)}{4 \sqrt{(1-z)\left(z+c_{-}\right)}} .
\end{gathered}
$$

Note that the domain of $g(z)$ is $z \in[-1,1)$ if $k \in[-2,0]$ and $z \in(2 k-1,1)$ if $k \in(0,1)$.

We observe that the fractional atomic imbalance $k$ plays an important role in the behavior of the $g(z)$ function. For $k \leqslant 0, g(z)$ is divergent only at $z=1$, while for the case of $k$ $>0, g(z)$ is divergent at $z=2 k-1$ and $z=1$. Since $k$ affects the domain and the shape of the function $g(z)$, this property will affect the type of solutions of Eq. (5). In Fig. 1 we illustrate the behavior of the function $g(z)$ for different values of $k$. It is, in fact, necessary to treat the cases of $k<0$, $k=0$, and $k>0$ separately.

\section{A. Negative case: $-\mathbf{2} \leqslant \boldsymbol{k}<0$}

Here the domain of $g(z)$ is $z \in[-1,1)$ and $g(z)$ is divergent at $z=1$, but finite at $z=-1$. This leads to the following classification for the solutions of Eq. (5):

(1) $\theta=0$ and $z$ is a solution of

$$
f(z)=g(z),
$$

which can admit zero, one, or two solutions.

(2) $\theta=N \pi / 6$ and $z$ is a solution of

$$
f(z)=-g(z),
$$

which can admit zero, one, or two solutions.

(3) $z=-c_{+}$, which vanishes the first equation of Eqs. (4) and reduces the second equation of Eqs. (4) to the expression

$$
\lambda=\frac{\alpha}{k+2}+\frac{\sqrt{-3 k(k+2)}}{2(k+2)} \cos \left(\frac{6 \theta}{N}\right),
$$

such that $\theta$ is a solution of

$$
\cos \left(\frac{6 \theta}{N}\right)=-\frac{2 \sqrt{-3 k(k+2)}}{3 k}\left(\lambda-\frac{\alpha}{k+2}\right),
$$

for which there are two solutions for $\left|\frac{2 \sqrt{-3 k(k+2)}}{3 k}\left(\lambda-\frac{\alpha}{k+2}\right)\right|<1$.

\section{B. Zero case: $k=0$}

Now we consider the case $k=0$, where the domain of $g(z)$ is $z \in(-1,1)$ and $g(z)$ is divergent at $z=1$, but finite at $z=-1$, similar to the previous case. This leads to the following classification for the general problem:

(1) $\theta=0$ and $z$ is a solution of

$$
f(z)=g(z),
$$

which can admit zero, one, or two solutions.

(2) $\theta=N \pi / 6$ and $z$ is a solution of

$$
f(z)=-g(z),
$$

which can admit zero, one, or two solutions.

(3) $z=-1$, which vanishes the first equation of Eqs. (4) and reduces the second equation of Eqs. (4) to the following linear equation between the coupling parameters:

$$
\lambda=\frac{\alpha}{2},
$$

which can admit just one solution. This result is compatible with that obtained in the previous case by taking the limit $k \rightarrow 0$ in Eq. (8).

\section{Positive case: $0<k \leqslant 1$}

In this case the domain of $g(z)$ is $z \in(2 k-1,1)$ and $g(z)$ is divergent at both extremes of the interval, $z=2 k-1$ and $z$ $=1$. Now, a different scenario emerges, compared to the previous two cases. This leads to the following classification for the general problem:

(1) $\theta=0$ and $z$ is a solution of

$$
f(z)=g(z),
$$

which can admit one, two, or three solutions.

(2) $\theta=N \pi / 6$ and $z$ is a solution of

$$
f(z)=-g(z),
$$

which can admit one, two, or three solutions.

We can collect all different types of solutions of Eq. (5) in a parameter diagram, dividing the parameter space into different regions, for each case of $k$ discussed above. For example, for the case of $k$ positive, to construct this diagram, we observe that the boundaries between each region occur when $f$ is the tangent line to $\pm g$; i.e., for values of $\lambda$ and $\alpha$ such that

$$
\begin{gathered}
\lambda= \pm\left.\frac{d g}{d z}\right|_{z_{0}}, \\
f\left(z_{0}\right)= \pm g\left(z_{0}\right),
\end{gathered}
$$

for some $z_{0}$. This requirement determines the boundaries in the parameter space, which are depicted in Fig. 2(c) for $k$ $=0.5$. 

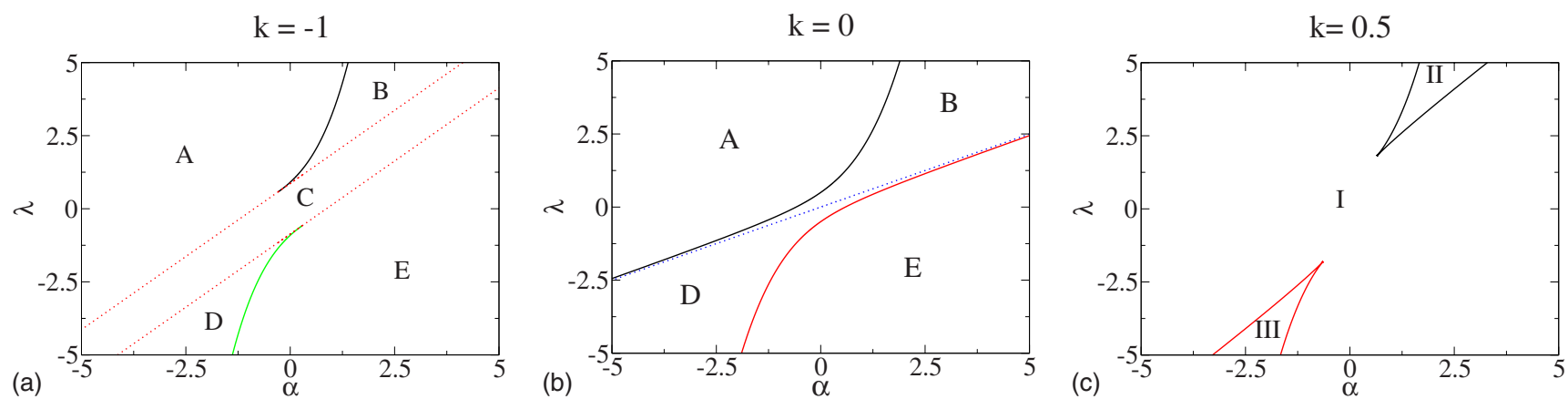

FIG. 2. (Color online) Parameter space diagram identifying the different types of solution for Eq. (5) for different values of $k=-1 ; 0 ; 0.5$. We observe (a) five distinct regions for the negative case; (b) four distinct regions for $k=0$; and (c) three distinct regions for the positive case. In (a) the boundaries are given by $\lambda=[\alpha \mp g(-k-1)] /(k+2)$ while in (b) it is given by $\lambda=\alpha / 2$.

As in the case of $k$ positive, we can determine the region boundaries in the parameter space for the other two cases. However, because of the existence of solutions of the form given by Eq. (8), which do not have an analog for positive $k$, we see the appearance of new boundaries given by the conditions $\lambda=[\alpha \mp g(-k-1)] /(k+2)$ for negative $k$ and $\lambda=\alpha / 2$ for $k=0$. The boundaries in parameter space are illustrated in Figs. 2(a) and 2(b) for $k=-1$ and $k=0$, respectively. Notice that the two additional boundaries, which delimit region $C$, for $k=-1$ are reduced to a unique boundary for $k=0$, which is not present for $k=0.5$. Therefore, we have a different scenario for the parameter space diagram, depending on if the fractional atomic imbalance $k$ is negative, zero, or positive, as illustrated in Fig. 2. Basically, we can summarize the typical behavior of the parameter space diagram as follows: when $k$ is negative, the parameter diagram is divided into five regions: in region $A$ there is no solution for $z$ when $\theta$ $=0$ and one solution for $z$ when $\theta=N \pi / 6$. In region $B$ there are two solutions for $z$ when $\theta=0$ and one solution for $z$ when $\theta=N \pi / 6$. In region $C$ there is one solution for $z$ when $\theta=0$, one solution for $z$ when $\theta=N \pi / 6$, and two solutions for $\theta$ when $z=-k-1$. In region $D$ there is one solution for $z$ when $\theta=0$ and two solutions for $z$ when $\theta=N \pi / 6$. In region $E$ there is one solution for $z$ when $\theta=0$ and no solution for $z$ when $\theta=N \pi / 6$. For the case $k=0$, region $C$ disappears and the phase diagram is left with the four regions $A, B, D$, and $E$ discussed before. When $k$ is positive the diagram is divided into three regions: in region I there is one solution for $z$ when $\theta=0$ and one solution for $z$ when $\theta=N \pi / 6$. In region II there are three solutions for $z$ when $\theta=0$ and one solution for $z$ when $\theta=N \pi / 6$. In region III there is one solution for $z$ when $\theta=0$ and three solutions for $z$ when $\theta=N \pi / 6$. It is interesting to mention that the fractional atomic imbalance also plays an important role in heterodiatomic molecular Bose-Einstein condensates [27-29].

To help visualize the classical dynamics, it is useful to plot the level curves of the Hamiltonian (3). Since the fixed points change the topology of the level curves, qualitative differences can be observed between the different regions. The results are depicted in Fig. 3 for $k=-1$ (on the left), $k$ $=0$ (in the middle), and $k=0.5$ (on the right). For clarity, we use convenient intervals for $\theta$ and $z$.

In Fig. 3(a) we show the level curves of the Hamiltonian (3) for $k=-1$, illustrating the typical behavior for regions $A$,
$B, C$, and $E$ (from the top to the bottom). In region $A$ there are local minima at $6 \theta / N= \pm \pi$. Besides the minima at $6 \theta / N= \pm \pi$, two additional fixed points (a maximum and a saddle point) are apparent in region $B$ occurring at $\theta=0$. In region $C$ there are minima at $6 \theta / N= \pm \pi$ and for $\theta=0$ just one fixed point, a maximum. There are also saddle points when $z=0$. In region $E$ just one fixed point, a maximum, occurs for $\theta=0$.

In Fig. 3(b) we show the level curves for $k=0$ for the same regions illustrated in the previous case, except that now instead of region $C$ there is just one straight line separating regions $B$ and $D$. The behavior here is analogous to the previous case of negative $k$, with the emergence of a maximum (minimum) when passing from region $A$ to $B$ ( $E$ to $C)$.

In Fig. 3(c) we present the level curves of the Hamiltonian (3) for $k=0.5$, illustrating the behavior of regions I, II, III and I (from the top to the bottom). In region I there is a maximal point at $\theta=0$ and a minima at $6 \theta / N= \pm \pi$. Two additional fixed points, a saddle and a maximum, occur in region II at $\theta=0$, while two additional fixed points, a saddle and a minimum, occur in region III at $6 \theta / N= \pm \pi$ compared to region $\mathrm{I}$.

We observe that the pattern of the level curves is distinct for the cases of $k$ negative and zero compared to the positive case.

In the following sections we will conduct an analysis of the quantum Hamiltonian. We will focus our attention on the case $\lambda=0$; in this way the model has one effective parameter, $\alpha$. In particular, we will establish that the bifurcation occurring at $(\alpha, \lambda)=(-g(-k-1), 0)$ for the negative case and $(\alpha, \lambda)=(0.5,0)$ for $k=0$ can be seen to influence the groundstate properties of the quantum system.

\section{QUANTUM ANALYSIS}

We now turn our attention to a quantum treatment of the model, to investigate the nature of the additional threshold couplings for the cases where the fractional atomic imbalance $k$ is negative and zero. In particular, we analyze the Hamiltonian in the no scattering limit where $U_{i j}=0$ for all $i, j=a, b, c$,

$$
H=\mu_{a} N_{a}+\mu_{b} N_{b}+\mu_{c} N_{c}+\Omega\left(a^{\dagger} a^{\dagger} b^{\dagger} c+c^{\dagger} b a a\right) .
$$



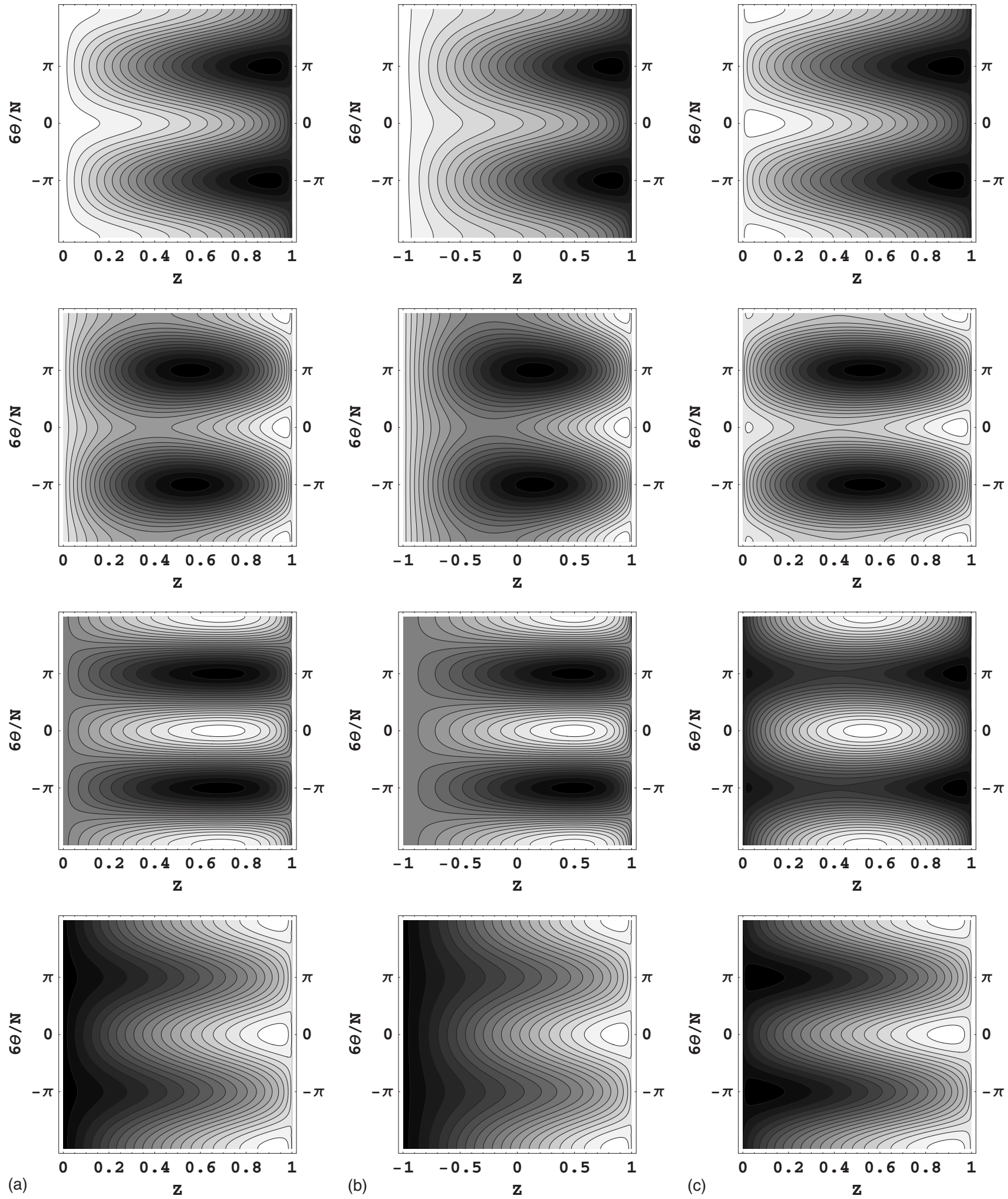

FIG. 3. Level curves for the Hamiltonian (3), where the dark regions indicate lower values than the light regions. Here we use for (a) $k=-1$ on the left, $(\lambda, \alpha)=(0 ;-1.0),(5 ; 2.5),(0,0)$, and $(0,1.5)$; (b) $k=0$ in the middle, $(\lambda, \alpha)=(0,-1),(2.5,2.5),(0 ; 0)$, and $(0,1.5)$; (c) $k=0.5$ on the right, $(\lambda, \alpha)=(0 ;-1.5),(5,2.5),(-5,-2.5)$, and $(0,1.5)$.

This simplifies substantially the Hamiltonian, however, it remains sufficiently nontrivial to enable us to gain an understanding of the quantum behavior through the quantum dynamics, ground-state expectation value, gap, and fidelity. The no scattering limit corresponds to the coupling $\lambda=0$ in the classical analysis of Sec. III. With reference to Fig. 2 there are two threshold couplings when $k$ is negative and three threshold couplings for $k=0$. For the case of $k$ negative, one 


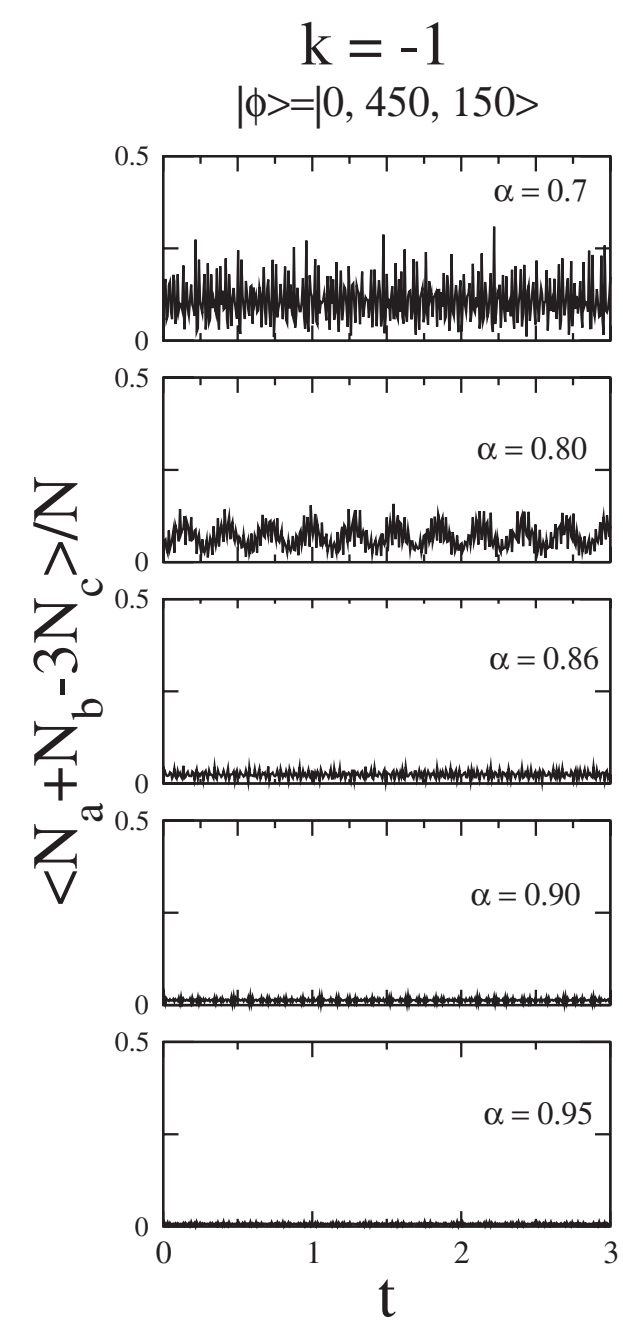

(a)

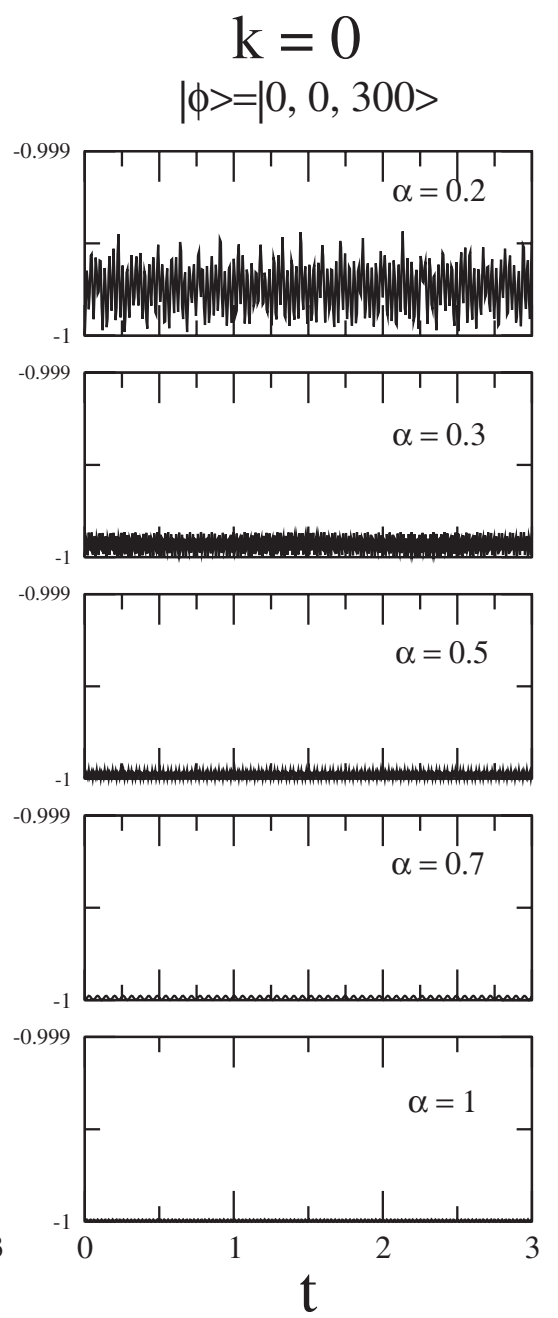

(b)
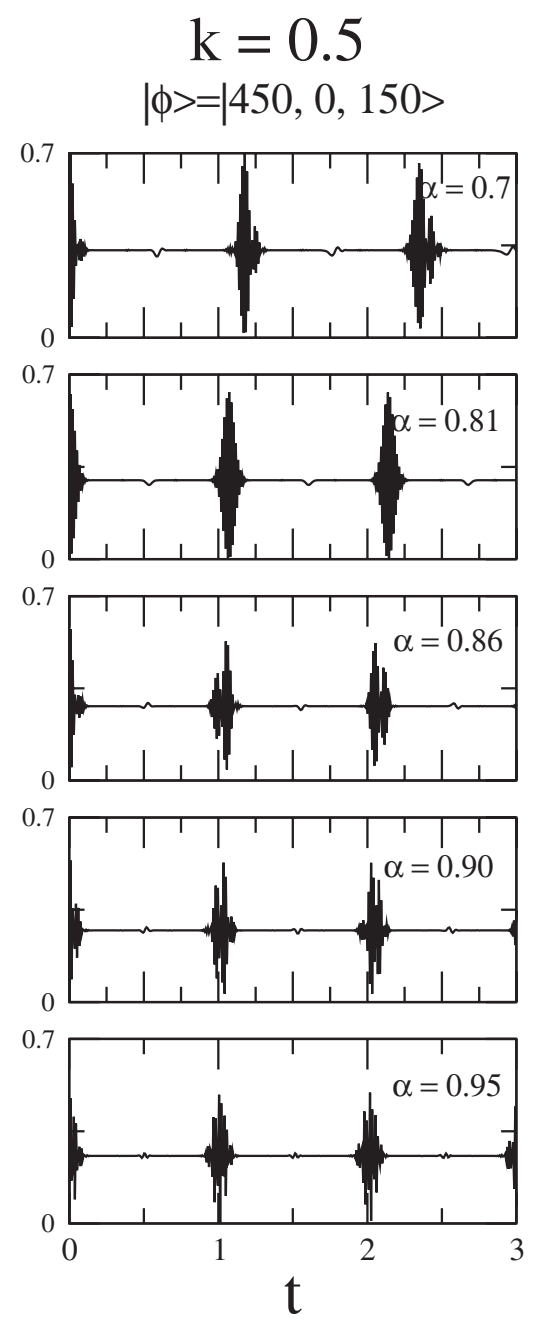

(c)

FIG. 4. Time evolution of the expectation value of $z$ for the Hamiltonian (15) with $N=900$, for (a) $k=-1$ and initial state $|0,450,150\rangle$. We are using natural units. The oscillations are largely irregular with significantly decreasing amplitude as the point at $\alpha=0.866$ is crossed. This point corresponds to the boundary at $(\alpha, \lambda)=(0.866,0)$ between regions $C$ and $E$ as shown in Fig. 2(a); (b) $k=0$ and initial state $\mid 0,0$, $300\rangle$. A similar behavior occurs as the point at $\alpha=0.5$ is crossed. This point corresponds to the boundary at $(\alpha, \lambda)=(0.5,0)$ as shown in Fig. 2(b); (c) $k=0.5$ with initial state $|450,0,150\rangle$. The oscillations display collapse and revival behavior with smoothly decreasing amplitude. Here there is no abrupt behavior, indicative of the fact there is no boundary at $\lambda=0$ in Fig. 2(c).

occurs at $(\alpha, \lambda)=(-g(-k-1), 0)$, signifying the bifurcation of the global minimum of the Hamiltonian, while the other occurs at $(\alpha, \lambda)=(g(-k-1), 0)$, signifying the bifurcation of the global maximum. For the specific example of $k=-1$, these thresholds are $(\alpha, \lambda)=(0.866,0)$ and $(\alpha, \lambda)=(-0.866,0)$, respectively. For the case $k=0$, there are three bifurcations at $(\alpha, \lambda)=(-0.5,0),(0,0),(0.5,0)$. The case $(\alpha, \lambda)=(-0.5,0)$ signifies the bifurcation of the global maximum, $(\alpha, \lambda)$ $=(0.5,0)$ signifies the bifurcation of the global minimum, while $(\alpha, \lambda)=(0,0)$ signifies the bifurcation of the saddle point. In contrast, there are no bifurcations along the line $\lambda$ $=0$ for the positive case. We focus our attention on the coupling $(\alpha, \lambda)=(0.866,0)$ for $k=-1$ and $(\alpha, \lambda)=(0.5,0)$ for $k$ $=0$, as in these cases the bifurcation of the fixed point in phase space is associated with the ground state of the quantum system.

\section{A. Quantum dynamics}

In general, the time evolution of any state is given by $|\Psi(t)\rangle=U(t)|\phi\rangle$, where $U(t)$ is the temporal operator, $U(t)$ $=\sum_{m=0}^{M}|m\rangle\left\langle m\left|\exp \left(-i E_{m} t\right),\right| m\right\rangle$ is an eigenstate with energy $E_{m}$, and $|\phi\rangle$ represents the initial state with $N=N_{a}+N_{b}$ $+3 N_{c}$. We adopt the method of directly diagonalizing the Hamiltonian (15) as done in [31,32] and compute the expectation value of $z(t)$ through

$$
\langle z(t)\rangle=\frac{1}{N}\left\langle\Psi(t)\left|N_{a}+N_{b}-3 N_{c}\right| \Psi(t)\right\rangle .
$$

In our analysis, for fixed total number of atoms $N$ and fixed atomic imbalance $J$, we will use the initial state configuration $|0,-J / 2,(2 N+J) / 6\rangle$ for the cases where $k$ is negative and zero and $|J, 0,(N-J) / 3\rangle$ for the case where $k$ is 


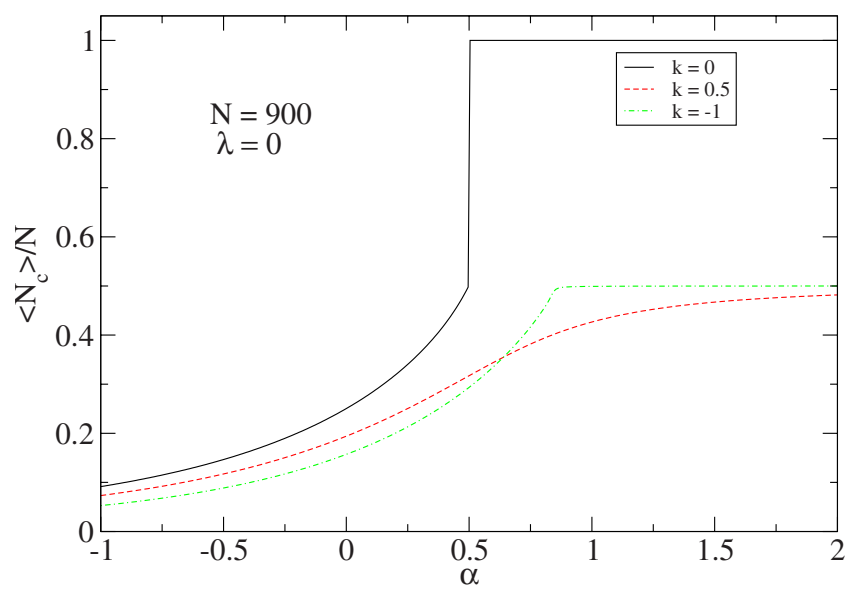

FIG. 5. (Color online) Normalized ground-state expectation value of the molecular number operator $\left\langle N_{c}\right\rangle$ versus the coupling parameter $\alpha$ for the three different cases $k=-1,0$, and 0.5 . Here we are using $\Omega=1$ and $N=900$. For the cases $k=-1$ and $k=0$ there is an abrupt change in the expectation value $3\left\langle N_{c}\right\rangle / N$ as the threshold coupling $\alpha=0.866$ (for $k=-1$ ) and $\alpha=0.5$ (for $k=0$ ) is reached. In contrast, for $k=0.5$, the expectation value $3\left\langle N_{c}\right\rangle / N$ increases smoothly with $\alpha$, not exhibiting any abrupt behavior.

positive. We therefore compare the three cases of the quantum dynamics with fractional atomic imbalance negative, zero, and positive.

Results of the expectation value for $z$ are shown in Fig. 4 for the cases of $k=-1,0$, and 0.5 . We are using $N=900$ and $J=-900 ; 0 ; 450$ for $k=-1 ; 0 ; 0.5$, respectively. We fix the parameter $\Omega=1$ and use $\mu_{c}$ as the variable coupling parameter. In terms of the classical variables, this corresponds to varying the parameter $\alpha$. The qualitative differences are quite apparent. In the case of $k=-1$, Fig. 4(a), we find that for $\alpha$ $<0.866$ there are irregular oscillations in $z$. Similar behavior occurs for $\alpha<0.5$ for $k=0$, Fig. 4(b). As the coupling parameter $\alpha$ is increased across the threshold value at $\alpha=0.866$, for $k=-1$ and $\alpha=0.5$ for $k=0$, the transition to localized oscillations is significant in cases (a) and (b). By comparison the dynamics in Fig. 4(c) for $k=0.5$ shows a collapse and revival of oscillations.

\section{B. Ground-state expectation values}

Now using Eq. (16), we compute the normalized groundstate expectation value $3\left\langle N_{c}\right\rangle / N$ for the quantum system as the coupling is varied. Results are shown in Fig. 5.

In general, agreement with the classical result is found: As the threshold coupling $\alpha=0.866$ (for $k=-1$ ) and $\alpha=0.5$ (for $k=0)$ is crossed, the maximal possible number of molecules that can be formed for each case $(100 \%$ for $k=-1$ and $50 \%$ for $k=0)$ is reached. In both cases, there is an abrupt change in the expectation value $3\left\langle N_{c}\right\rangle / N$ at the threshold point. However, for $k=0$, the expectation value $3\left\langle N_{c}\right\rangle / N$ does not exhibit any sudden change, indicative of the fact that there is no boundary in Fig. 2(c). Therefore, qualitative changes are observed between the cases of $k$ negative and zero and the case of $k$ positive.

\section{Quantum phase transitions}

In order to gain better insight into the effect of the threshold couplings for the quantum system, in our final analysis we investigate the existence of quantum phase transitions in our model (15).

A quantum phase transition (QPT) is usually defined as a phase transition in the ground state of the system under the variation of some parameter. Basically, there is a sudden change in the structure of the ground state at the QPT, and the properties such as entanglement, correlations, etc. reflect this sudden change [30]. There are different methods to determine a QPT. In particular, we will study the behavior of the energy gap and fidelity of the system to identify a QPT. Here we mention that a QPT is rigorously defined in the thermodynamical limit $N \rightarrow \infty$. For large but finite $N$ the system does display an increasingly sharp distinction between ground-state regions, called quantum prephase transitions (QPPT). The occurrence of a QPPT in a finite system is a precursor for a QPT in the thermodynamic limit. Let us now study the QPPT of the Hamiltonian (15).

\section{Energy gap}

One possibility to identify a QPPT is through the energy gap, which is defined as the difference between the first excited state and the ground state of the system.

$$
\Delta E=E_{1}-E_{0} .
$$

In Fig. 6 we plot the gap against the coupling parameter $\alpha$ for the cases of $k=-1,0$, and 0.5 using $\Omega=1$ and different values of $N$. In all cases the energy gap exhibits a minimum,
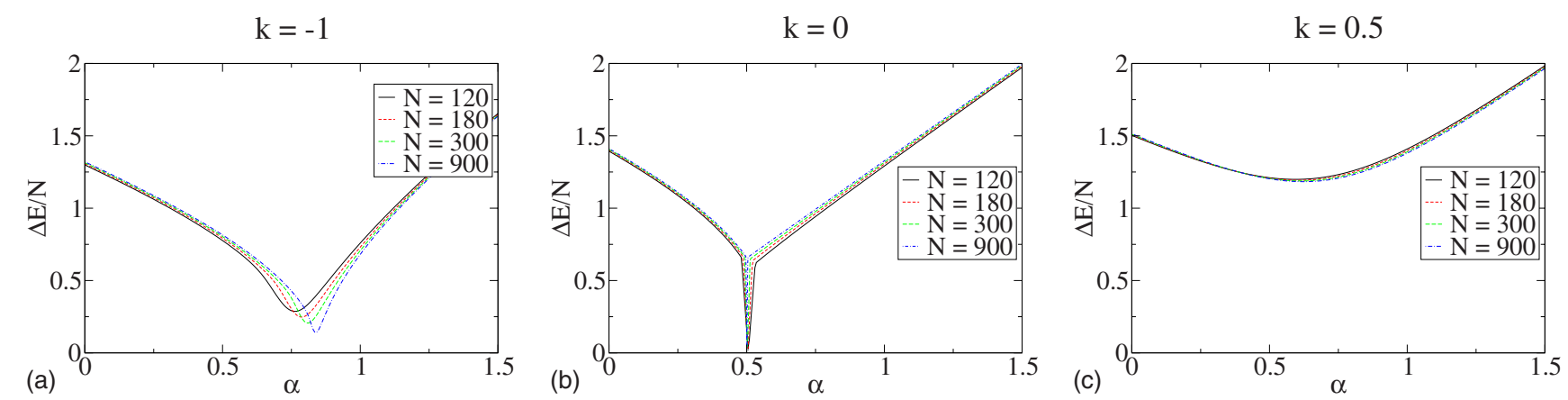

FIG. 6. (Color online) Energy gap between the first excited state and the ground state as a function of $\alpha$ for (a) $k=-1$, (b) $k=0$, and (c) $k=0.5$ and different values of $N$. We are using $\Omega=1$ and natural units. 

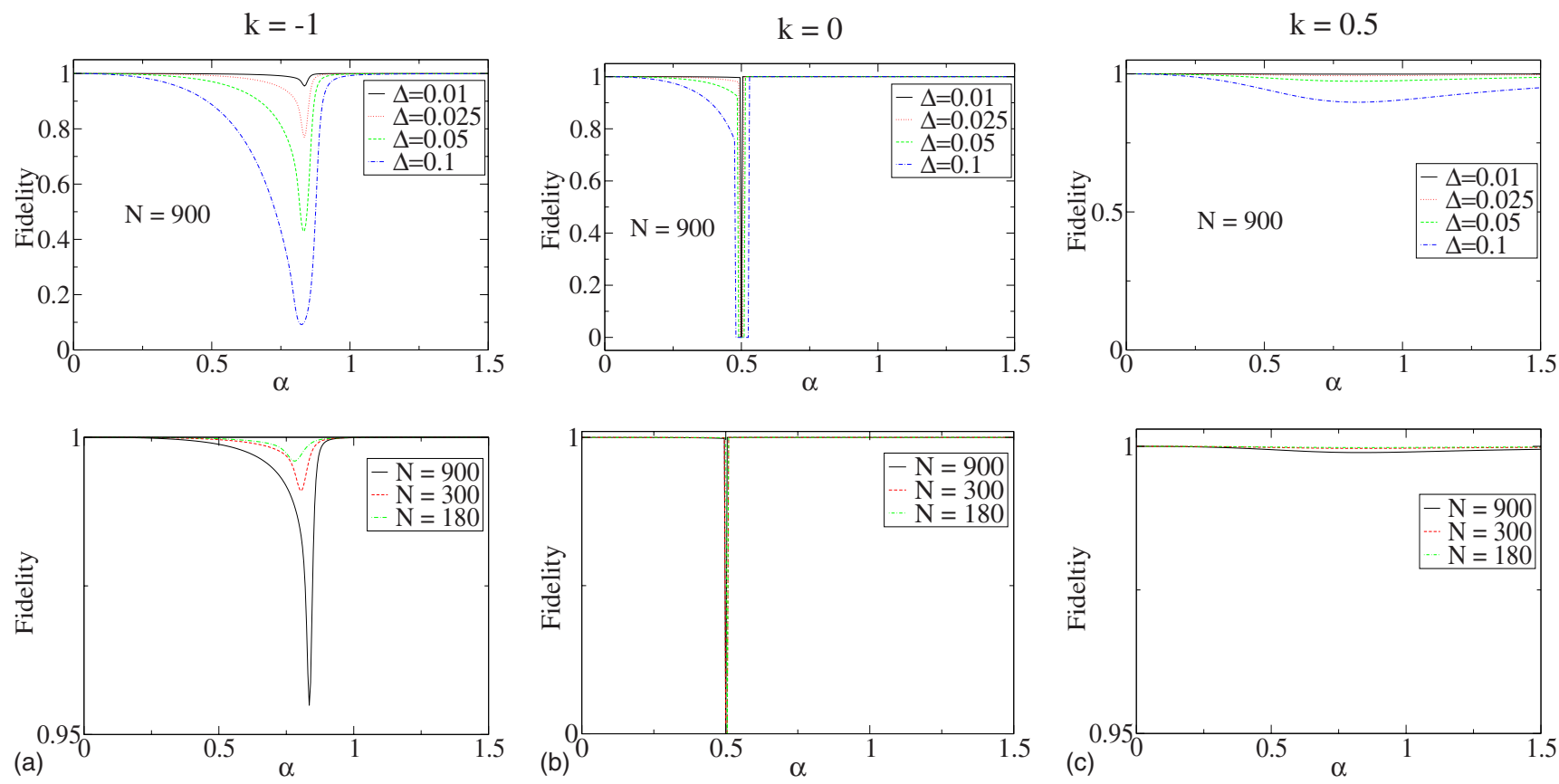

FIG. 7. (Color online) Ground-state wave-function overlaps as a function of the coupling parameter $\alpha$ for (a) $k=-1$, (b) $k=0$, and (c) $k=0.5$ and $\Omega=1$. On the top we are using $N=900$ and different values of $\Delta$. In the bottom we are using $\Delta=0.01$ and different values of $N$. In all cases the fidelity exhibits a minimum, which is substantially more pronounced for $k=-1$ and $k=0$, compared to $k=0.5$.

which is much more pronounced in the cases of $k$ negative and zero compared to the case where $k$ is positive. We observe that as long as $N$ increases, the point where the gap tends to vanish corresponds to $\alpha=0.866$ for $k=-1$ [Fig. 6(a)] and $\alpha=0.5$ for $k=0$ [Fig. 6(b)], in agreement with the classical analysis. In contrast, when $k$ is positive there is no abrupt variation of the energy gap as shown in Fig. 6(c) and QPPT are not expected.

\section{Fidelity}

Another possibility to investigate the QPPT is through the behavior of the fidelity, which is a concept widely used in the quantum information theory [33-35]. The fidelity is basically defined as the modulus of the wave-function overlap between two quantum states. Assuming the ground state of the system is nondegenerate, let $\Psi(\alpha)$ denote the unique normalized ground state. For fixed small $\Delta$ we define the function fidelity or ground-state wave-function overlap $\mathcal{F}_{\Delta}(\alpha)$ by

$$
\mathcal{F}_{\Delta}(\alpha)=|\langle\Psi[\alpha(1-\Delta)] \mid \Psi[\alpha(1+\Delta)]\rangle|,
$$

which is symmetric in $\Delta$, bounded between 0 and 1 , and satisfies $\mathcal{F}_{0}(\alpha)=1$. For systems that exhibit a quantum phase transition in the thermodynamic limit, the wave-function overlaps between states in different phases go to zero in this limit. The occurrence of a minimum in the ground-state wave-function overlap in a finite system is then a precursor for a quantum phase transition in the thermodynamic limit. Thus for finite systems we identify quantum phase pretransitions at a coupling $\alpha$ for which the fidelity is (locally) minimal. Figure 7 shows the behavior of the fidelity for (a) $k=-1$, (b) $k=0$, and (c) $k=0.5$ for fixed $N(\Delta)$ and different values of $\Delta(N)$ on the top (bottom). It is clear that the minimum value of $\mathcal{F}_{\Delta}(\alpha)$, which determines the quantum phase pretransition, tends to occur at $\alpha \approx 0.86$ for $k=-1$ and $\alpha$ $\approx 0.5$ for $k=0$. The distinction between the predicted threshold coupling and the observed pretransition coupling is that the pretransition coupling also occurs for $k$ positive, although for fixed $N$ the minimum of $\mathcal{F}_{\Delta}(\alpha)$ is substantially more pronounced for $k$ negative and zero compared to $k$ positive. In all instances the value of the minimum decreases with increasing $N$. We remark that the value of $\alpha$ at which the minimum occurs is independent of $\Delta$, as shown in Fig. 7 (on the top). In our previous classical analysis qualitative differences are only found precisely when $k$ is negative or zero. Here it is clear that the distinguishability of two phases is more reliable also for $k$ negative or zero. We then interpret these results as the emergence of quantum phase boundaries for $k$ negative or zero.

\section{CONCLUSION}

We have considered a model describing a mixture of two species of atoms in different proportions, which can combine to form a bound molecular state at zero temperature. This heterotriatomic molecular Bose-Einstein condensate model has been investigated in detail through a classical and a quantum analysis.

We have found that the fractional atomic population imbalance $k$, an extra control "knob" characteristic to hetero- 
nuclear models, plays an important role in the determination of the phase boundaries in the diagram of parameters in the classical analysis. This property also holds at a quantum level by inspecting the ground-state expectation values and the character of the quantum dynamics of the model. We have also looked for the quantum phase pretransitions in our system and shown that the quantities energy gap and groundstate fidelity are suited for revealing QPPT and pinning down the critical (bifurcation) points.

\section{ACKNOWLEDGMENTS}

C.C.N.K. and A.F. thank J. Links and E. Mattei for discussions. A.P.T., A.F., I.R., and Z.V.S.T. would like to thank CNPq-Conselho Nacional de Desenvolvimento Científico e Tecnológico for financial support. I.R. also thanks FAPERJFundação Carlos Chagas Filho de Amparo à Pesquisa do Estado do Rio de Janeiro for financial support. G.S. would like to thank CAPES-Coordenação de Aperfeiçoamento de Pessoal de Nível Superior for financial support.
[1] E. A. Cornell and C. E. Wieman, Rev. Mod. Phys. 74, 875 (2002).

[2] J. R. Anglin and W. Ketterle, Nature (London) 416, 211 (2002).

[3] D. J. Heinzen, R. Wynar, P. D. Drummond, and K. V. Kheruntsyan, Phys. Rev. Lett. 84, 5029 (2000).

[4] P. Zoller, Nature (London) 417, 493 (2002).

[5] S. B. Papp and C. E. Wieman, Phys. Rev. Lett. 97, 180404 (2006).

[6] G. Thalhammer, G. Barontini, L. De Sarlo, J. Catani, F. Minardi, and M. Ignuscio, Phys. Rev. Lett. 100, 210402 (2008).

[7] J. Catani, L. De Sarlo, G. Barontini, F. Minardi, and M. Inguscio, Phys. Rev. A 77, 011603(R) (2008).

[8] B. Damski, L. Santos, E. Tiemann, M. Lewenstein, S. Kotochigova, P. Julienne, and P. Zoller, Phys. Rev. Lett. 90, 110401 (2003).

[9] J. Herbig, T. Kraemer, M. Mark, T. Weber, C. Chin, H.-C. Nagerl, and R. Grimm, Science 301, 1510 (2003).

[10] T. Weber, J. Herbig, M. Mark, H.-C. Nagerl, and R. Grimm, Phys. Rev. Lett. 91, 123201 (2003).

[11] T. Kraemer, M. Mark, P. Waldburger, J. G. Danzl, C. Chin, B. Engeser, A. D. Lange, K. Pilch, A. Jaakkola, H.-C. Nagerl, and R. Grimm, Nature (London) 440, 315 (2006).

[12] H.-Q. Zhou, J. Links, M. Gould, and R. McKenzie, J. Math. Phys. 44, 4690 (2003).

[13] J. Links, H.-Q. Zhou, R. H. McKenzie, and M. D. Gould, J. Phys. A 36, R63 (2003).

[14] H.-Q. Zhou, J. Links, R. H. McKenzie, and X.-W. Guan, J. Phys. A 36, L113 (2003).

[15] A. Foerster, J. Links, and H.-Q. Zhou, in Classical and Quantum Nonlinear Integrable Systems: Theory and Applications, edited by A. Kundu (IOP Publishing, Bristol and Philadelphia, 2003), pp. 208-233.

[16] J. Dukelsky, G. G. Dussel, C. Esebbag, and S. Pittel, Phys.
Rev. Lett. 93, 050403 (2004).

[17] G. Ortiz, R. Somma, J. Dukelsky, and S. Rombouls, Nucl. Phys. B 707, 421 (2005).

[18] A. Kundu, Theor. Math. Phys. 151, 831 (2007).

[19] A. Foerster and E. Ragoucy, Nucl. Phys. B 777, 373 (2007).

[20] J. Li, D.-F. Ye, C. Ma, L.-B. Fu, and J. Liu, e-print arXiv:0807.1691.

[21] X.-J. Liu, H. Hu, and P. D. Drummond, Phys. Rev. A 77, 013622 (2008).

[22] M. Héritier, Nature (London) 414, 31 (2001).

[23] M. T. Batchelor, Phys. Today 60 (1), 36 (2007).

[24] F. Ferlaino, S. Knoop, and R. Grimm, e-print arXiv:0809.3920.

[25] J. Catani (private communication).

[26] G. Santos, A. Foerster, I. Roditi, Z. Santos, and A. Tonel, J. Phys. A: Math. Theor. 41, 295003 (2008).

[27] M. Duncan, A. Foerster, J. Links, E. Mattei, N. Oelkers, and A. Tonel, Nucl. Phys. B 767, 227 (2007).

[28] L. Zhou, J. Qian, H. Pu, W. Zhang, and H. Y. Ling, Phys. Rev. A 78, 053612 (2008).

[29] L. Zhou, W. Zhang, H. Y. Ling, L. Jiang, and H. Pu, Phys. Rev. A 75, 043603 (2007).

[30] S. Sadchev, Quantum Phase Transitions (Cambridge University Press, Cambridge, England, 2001).

[31] A. P. Tonel, J. Links, and A. Foerster, J. Phys. A 38, 1235 (2005).

[32] A. P. Tonel, J. Links, and A. Foerster, J. Phys. A 38, 6879 (2005).

[33] M. A. Nielsen and I. L. Chuang, Quantum Computation and Quantum Information (Cambridge University Press, Cambridge, England, 2000).

[34] H-Q. Zhou and J. P. Barjaktarevic, J. Phys. A 41, 412001 (2008).

[35] P. Buonsante and A. Vezzani, Phys. Rev. Lett. 98, 110601 (2007). 